\title{
Dynamic multimodal full-field optical coherence tomography and fluorescence structured illumination microscopy
}

\author{
Olivier Thouvenin \\ Mathias Fink \\ Claude Boccara
}




\title{
Dynamic multimodal full-field optical coherence tomography and fluorescence structured illumination microscopy
}

Olivier Thouvenin, Mathias Fink, and Claude Boccara*

PSL Research University, Institut Langevin, ESPCI Paris, Paris, France

\begin{abstract}
We report on the development of a configuration of a multimodal full-field optical coherence tomography (FF-OCT) and fluorescence microscope. Our system can simultaneously acquire FF-OCT and structured illumination microscopy images. Dynamic parallel evolution of tissue microstructures and biochemical environments can be visualized. We use high numerical aperture objectives to optimize the combination of the two modalities. We imaged the propagation of mechanical waves initiated by calcium waves in a heart wall to illustrate the interest of simultaneous recording of mechanical and biochemical information. $\odot$ The Authors. Published by SPIE under a Creative Commons Attribution 3.0 Unported License. Distribution or reproduction of this work in whole or in part requires full attribution of the original publication, including its DOI. [DOI: 10.1117/1.JBO.22.2.026004]
\end{abstract}

Keywords: full-field optical coherence tomography; structured illumination microscopy; multimodality; tomography.

Paper 160770R received Nov. 15, 2016; accepted for publication Jan. 18, 2017; published online Feb. 14, 2017.

\section{Introduction}

Optical coherence tomography (OCT) is widely used in the biomedical field to image microstructural morphology in depth in tissue without labeling. ${ }^{1,2}$ Full-field optical coherence tomography (FF-OCT) is a full-field, high transverse resolution version of OCT. ${ }^{3,4}$ It uses simple incoherent light sources, high numerical aperture (NA) objectives, and two-dimensional detectors to acquire en-face views of the sample at a given depth without scanning. FF-OCT records the backreflected and backscattered light coming from a defined volume of the sample. Due to its submicron resolution in three-dimensions (3-D), FF-OCT can reveal structures such as myelinated axons in brain samples, ${ }^{5}$ collagen fibers in liver, and blood vessel walls. ${ }^{6}$ It can also reveal subcellular contrast as it can image the position of the nucleus and the inner structures of a cell. ${ }^{7}$

Recently, new techniques, based either on OCT or FF-OCT, have enabled the study not only of the static 3-D morphology of a sample but also of the dynamics of its scatterers. ${ }^{7,8}$ In ex vivo tissues, these dynamic techniques can add new contrast based on the intracellular motility. ${ }^{9}$

OCT techniques can also detect collective motion of scatterers, resulting from propagating elastic waves. Measuring the speed of such waves allows measurement of the elasticity tissue, down to the microstructural level. ${ }^{10}$

For dynamic measurements and wave imaging, OCT techniques have two major advantages. First, their sectioning ability enables exclusion of scatterers movements outside the coherence volume. Second, their interferometric nature makes them sensitive to phase changes in the axial dimension that are much smaller than the optical axial resolution, down to a dozen nanometers. To summarize, OCT techniques, and FF-OCT in particular due to its higher resolution, enable

*Address all correspondence to: Claude Boccara, E-mail: claude.boccara@ espci.fr noninvasive visualization of biological tissue microstructural morphology and dynamics.

However, OCT techniques are limited in their identification of specific molecular structures. OCT images often need to be compared with fluorescence or histology measurements to understand and identify the observed microstructures using additional specific markers.

On the other hand, fluorescence microscopy can reveal very specific molecular contrast using chemical dyes or genetically encoded proteins that can be attached to a specific structure of interest. It can also take advantage of dynamic fluorophores and observe fluorescence fluctuations caused by changes of the biochemical properties of the tissue. For example, calcium imaging is very efficient for quantifying calcium uptake inside specific cells. ${ }^{11,12}$ Other indicators can detect changes in cell membrane voltage ${ }^{13}$ or in the ATP concentration inside the cell, ${ }^{14}$ and many more are currently being developed. Accurate fluorescence measurements can be performed inside biological tissues using optical techniques that provide optical sectioning. Among them, structured illumination microscopy (SIM) can be described as a full-field version of confocal microscopy and can perform optical sections as thin as a few micrometers in a tissue. $^{15-17}$

However, with fluorescence microscopy, it is often hard to understand the positioning of labeled structures of interest in the context of the surrounding tissue or their interaction with neighboring biological structures. Even if multiple labeling can help to overcome this problem, it strongly increases the complexity of the experiments, and ideally, a different label should be used for every structure of interest. Moreover, obtaining homogeneous and efficient labeling is often complex, expensive, and time consuming. Finally, photobleaching and phototoxicity can be problematic for long-term measurements.

Therefore, combining OCT and fluorescence in a single optical setup can overcome several difficulties of each modality operated separately and can provide new biological information. The interest of combining fluorescence imaging and OCT has 
been demonstrated in different publications. ${ }^{18-21}$ Due to its fullfield configuration, SIM is the most natural 3-D fluorescence technique to couple with FF-OCT. One major interest of such multimodal setups is to provide in situ validation of the identity of structures observed with (FF-)OCT to dispense with labeling in subsequent steps. Once a structure or cell type is confirmed with fluorescence, it can be identified directly in future OCT images, without labeling. Viewing with both modalities in overlay helps reduce ambiguity in structure identification that could arise from sequential imaging with two separate microscopes. ${ }^{22}$ Finally, FF-OCT can detect myelinated fibers due to their high refractive index mismatch, producing a strong specular reflection. ${ }^{5}$ It is particularly interesting since, to our knowledge, there is no specific fluorescent label for myelin, except in mice, where a genetic line has been developed.

In this paper, we report on the development and different applications of a setup combining FF-OCT and SIM. A similar combination has already been described; $;^{20,21}$ however, these setups used a flip mirror to switch sequentially from one modality to the other. In contrast, our setup can simultaneously acquire images from both modalities, which is key when imaging dynamic events. Simultaneous operation also helps to reduce acquisition times, which can be critical when large 3-D stacks are required.

Makhlouf et al. ${ }^{23}$ described a simultaneous static FF-OCT and fluorescence approach similar to ours. In our paper, we demonstrate the interest of adding a third modality by associating static and dynamic full-field OCT and fluorescence measurement synchronously in time and space. We developed different biological imaging applications in various contexts, including the embryology, ophthalmology, and cardiology fields.

We also demonstrate that using high NA objectives can facilitate the association of both techniques. Increasing the NA not only improves the transverse resolution and the photon collection, it also imposes a low longitudinal spatial coherence and a thin coherence volume that can take over from the temporal coherence volume. ${ }^{24}$ Due to high NA objectives, the optical section can therefore be controlled by the angular distribution created by the objective, and thin optical sections can be obtained even with narrowband sources, which is key to preventing spectral overlap between FF-OCT and fluorescence signals and, in turn, increases the setup versatility.

\section{Methods}

\subsection{Full-Field Optical Coherence Tomography}

Our experimental setup is shown in Fig. 1(a). It is based on a Linnik interference microscope configuration. To perform FF-OCT measurements, the microscope is illuminated by a temporally and spatially incoherent light source. In practice, we used a high power red LED (Thorlabs M660L3, nominal wavelength: $660 \mathrm{~nm}$, spectral bandwidth: $20 \mathrm{~nm}$ ) mounted in a pseudo Köhler configuration. In contrast to a standard Köhler configuration, we used only one lens in the illumination path to save light and build a more compact system. The aperture diaphragm is directly conjugate with the back focal plane of the objective, while the field diaphragm is at the focal plane of the single lens and is, therefore, imaged at the focal plane. The source is mounted as closely as possible to the aperture diaphragm to remove the spatial heterogeneity of the source. A 90:10 visible cube beamsplitter splits the light into sample and reference arms. Each arm contains an identical $40 \times$ water immersion microscope objective (Nikon CFI APO 40× water NIR objective, $0.8 \mathrm{NA}, 3.5 \mathrm{~mm}$ working distance).

In the reference arm, light is focused onto a flat silicon wafer that gives a reflection coefficient of about $23.5 \%$ at the interface with water. The reference arm length can be adjusted using a motorized one-dimensional translation stage.

In the sample arm, FF-OCT detects any structure that either reflects or backscatters the light. The backscattered and reflected coefficients depend on the refractive index and the size and shape of the imaged structures. ${ }^{25}$ A manual $X Y$ translation stage is attached to the sample objective for symmetry purpose. A second manual $X Y$ and a motorized $Z$ translation stage are used to move the sample independently of the sample objective.

Light returning from both arms is recombined by the entrance beamsplitter. The two beams interfere only if the optical path length difference between both arms remains within the coherence length of the system.

A $25-\mathrm{cm}$ focal length achromatic doublet focuses the light to a high speed and high full well capacity CMOS Camera (PhotonFocus, MV-D1024E-160-CL-12). The overall magnification of the FF-OCT path is therefore $50 \times$.

A piezoelectric actuator modulates the position of the reference mirror in four consecutive positions separated by $\lambda / 4$. Then, complex amplitude and phase images are computed from these four consecutive frames.

Transverse resolution and depth of field are determined by the microscope objective. With the objectives we used, we measured a transverse resolution of $0.525 \mu \mathrm{m}$ and an axial resolution of $1.5 \mu \mathrm{m}$, close to the expected theoretical resolutions. These parameters were measured using the point spread function produced by imaging gold nanobeads of $100 \mathrm{~nm}$ diameter.

Since FF-OCT acquires en-face images on the camera, the frame rate is limited only by the camera frame rate. Our camera can acquire $1024 \times 1024$ pixels images at $137 \mathrm{~Hz}$ and can acquire images at more than 1000 images/s for reduced fields of view. As amplitude and phase images require four direct images to be computed, the actual acquisition speed of static FF-OCT is $34 \mathrm{~Hz}$.

The principle of FF-OCT is to use an incoherent light source to localize the interference only to where optical path difference is close to 0 . In practice, the optical section gives the thickness over which the interference signal can be detected. The thickness of the optical section is determined by the coherence length of the microscope, which can be controlled either by the temporal coherence of the source or by the spatial coherence of the system.

In our case, we used an FF-OCT source with a rather narrow spectral band to avoid the spectral overlap with the fluorescence light. Therefore, the temporal coherence length of the system has been measured to be only $7.75 \mu \mathrm{m}$ [Fig. 1(d)]. However, using high NA objectives also enables confinement of the interference signal. ${ }^{26,27}$ Qualitatively, two beams can interfere only if their wavefronts have similar shapes. Therefore, light backscattered by an out of focus structure is not able to interfere with the perfectly focused beam of the reference arm. Interestingly, this longitudinal spatial incoherence can even be observed with a spatially coherent light, ${ }^{26,27}$ since the source of spatial incoherence mainly comes from the large angular distribution offered by the high NA objective. ${ }^{24}$ Qualitatively, using high NA objectives creates a spatial coherence gate of the size of the depth of field of the objective. The total coherence 


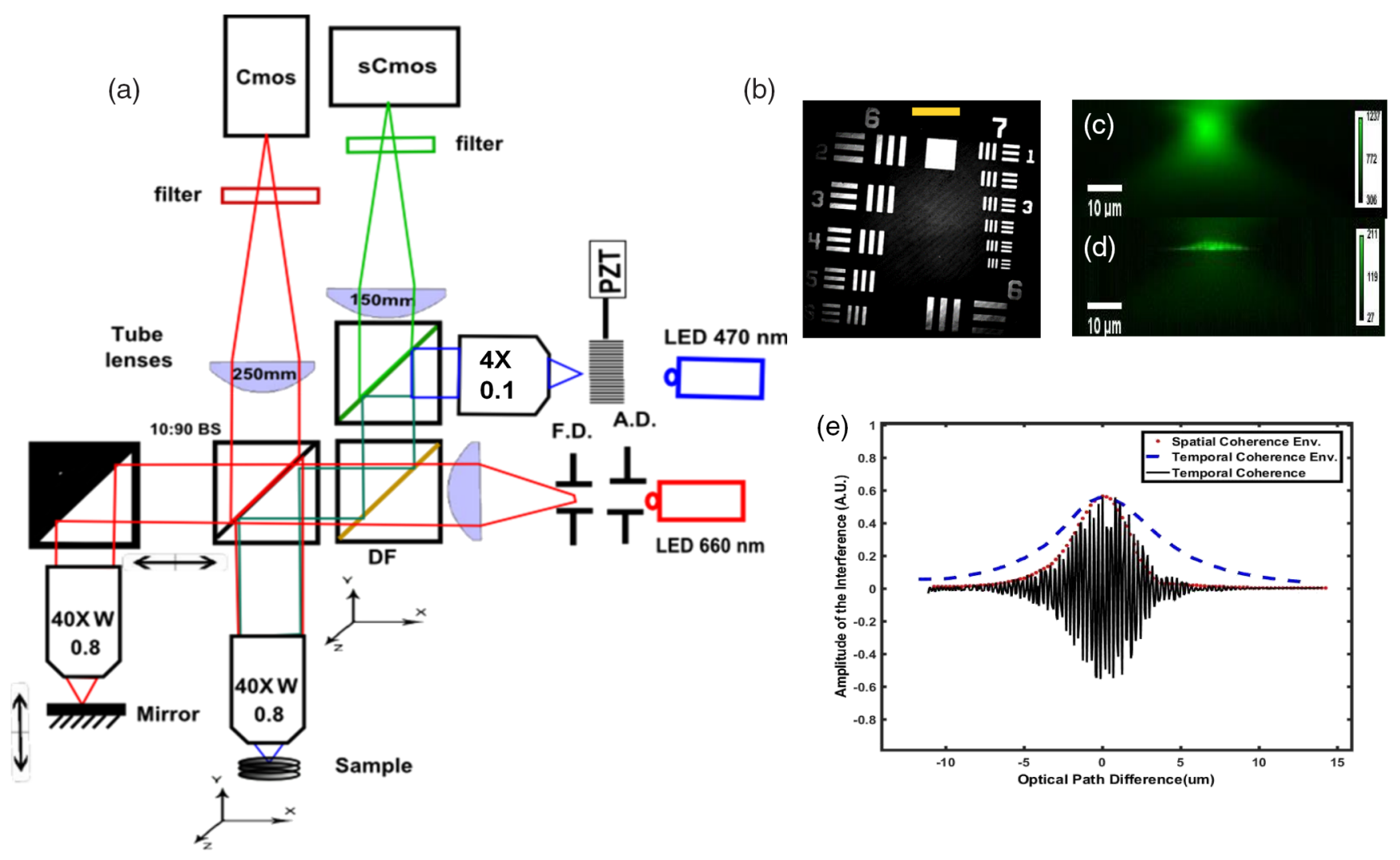

Fig. 1 Setup schematic and characterization. (a) Synchronous multimodal setup associating FF-OCT and SIM. The light path presented in red is the FF-OCT path, which consists of a Linnik interferometer with a 90:10 beamsplitter, two 40×, 0.8 NA objectives, and red LED $(660 \pm 10 \mathrm{~nm})$ pseudo-Köhler illumination. F.D. and A.D. stand for field and aperture diaphragms, respectively. A DF at $595 \mathrm{~nm}$ separates the FF-OCT path from the fluorescence path, represented in blue and green on the schematic. A blue LED $(470 \pm 13 \mathrm{~nm})$ shines onto a Ronchi ruling with a frequency of 100 lines per $\mathrm{mm}$. The Ronchi ruling is imaged with a $4 \times, 0.1 \mathrm{NA}$ objective and positioned conjugate to the focal plane of the imaging objectives. The grating period is $1.1 \mu \mathrm{m}$ in the sample plane. Fluorescence from the sample arm is captured by the sample objective and reflected by the interferometer beamsplitter and the DF. A second DF at $500 \mathrm{~nm}$ separates excitation from emission light, and fluorescence is imaged onto a sCMOS camera. Tube lenses from FF-OCT and SIM paths are 250 and $150 \mathrm{~mm}$, respectively. (b) An USAF target is imaged to illustrate the high transverse resolution of the microscope. The yellow scale bar in (b) represents $50 \mu \mathrm{m}$. (c, d) Illustrations of the sectioning ability of SIM. A rat hippocampal cultured neuron tagged with Fluo-4 is imaged without SIM in (c) and with SIM in (d). In (c, d), the $X Z$ profile of the average fluorescence collected on $10 \mu \mathrm{m}$ is displayed. The smallest resolvable object in SIM is measured at $2.9 \mu \mathrm{m}$, which is supposedly larger than the smallest compartments of a neuron. Thus, we estimate the size of the SIM optical section to be equal to $2.9 \mu \mathrm{m}$. (e) Sectioning capability of the setup. All curves are obtained by looking at interference fringes produced by placing similar silicon wafers in both arms. The black and red curves represent the intensity modulation and the fringe envelope, respectively, obtained by changing the focus of the sample arm to probe the spatial coherence of the system. ${ }^{24}$ The full width at half maximum of the envelopes, corresponding to the spatial and temporal optical sections, were measured to be 4.0 and $7.75 \mu \mathrm{m}$.

gate of the FF-OCT microscope is mainly controlled by the thinner coherence gate between the spatial and the temporal coherence gates, especially when one of the coherence gates is large compared to the other. ${ }^{24}$ In our microscope, we measured our final coherence gate to be $4.0 \mu \mathrm{m}$ thick, as illustrated in Fig. 1(d).

To experimentally measure the temporal and spatial optical sections offered by our microscope, we measured the decay of fringe contrast using two different scanning schemes. The interference fringes are obtained by placing a flat silicon wafer in both arms.

To measure the temporal coherence gate, we first performed a P-scan by scanning the length of the reference arm, without changing the focus of the two objectives. ${ }^{24}$ This procedure only scans the optical path difference without changing the beam wavefronts, so the coherence volume is only controlled by the temporal coherence of the system. To measure the overall coherence gate, we performed a standard Z-scan of the sample arm. As the silicon wafer is scanned along the axial dimension, this not only modulates the optical path difference but also adds a defocus to the wavefront, preventing the formation of interference fringes. The results of such scans are presented in Fig. 1(d), and we observe that the total optical section is $4.0 \mu \mathrm{m}$ thick instead of the $7.75 \mu \mathrm{m}$ expected from the temporal coherence.

Finally, even though the use of a spatially incoherent light is not required to reduce the optical section, it suppresses cross talk $^{28}$ and preserves the transverse resolution from degradation caused by aberrations. ${ }^{29}$ 


\subsection{Structured Illumination Fluorescence Microscopy}

In the current configuration, we used a high power blue LED (Thorlabs, M470L3, nominal wavelength: $470 \mathrm{~nm}$, spectral bandwidth: $25 \mathrm{~nm}$ ) filtered with a bandpass filter centered around $480 \mathrm{~nm}$ to excite fluorophores. The LED shines onto a grid of 100 lines/mm imaged by an air objective (Olympus $4 \times, 0.1 \mathrm{NA})$ and conjugated to the sample image plane. The light coming out of the objective is made collinear with the FF-OCT beam because of a dichroic mirror centered at $595 \mathrm{~nm}$. The light then enters the interferometer, but fluorescence is collected only from the sample arm. Then, a longpass dichroic filter (DF) centered at $595 \mathrm{~nm}$ reflects the fluorescence signal onto an achromatic doublet of focal length $150 \mathrm{~mm}$. The latter focuses the light onto a high-speed low-noise sCMOS camera (PCO.edge 5.1). Therefore, the overall magnification of the fluorescence path is $30 \times$ instead of the $50 \times$ of the FFOCT path. Using different magnifications enables us to have similar imaged pixel sizes from both modalities, even though the physical size of the pixels is different for each camera.

To reconstruct optically sectioned images, we designed an SIM by conjugating a physical grid (Ronchi ruling) with the sample plane. We mounted the grid on a piezo-driven translation stage. Because the grid pattern is only convolved with fluorescent structures that are at the sample plane, it becomes possible to numerically exclude out-of-focus fluorescence by translating the grid and excluding static signals. To translate the grid, we mounted the Ronchi ruling on a piezo-driven stage. Even though only three images are required to reconstruct a fluorescence image, we often acquire four images. It helps to reduce grid artifacts, but more importantly, it enables matching of SIM and FF-OCT acquisition rates, with similar exposure times for both modalities.

The grid frequency is 100 lines $/ \mathrm{mm}$, which corresponds to a distance between two lines of $1.1 \mu \mathrm{m}$ in the sample plane, so the SIM optical section is $2.5 \mu \mathrm{m}$ thick. ${ }^{15}$ Again, a trade off needs to be made between the size of the optical section and the visibility of the grid pattern. The higher the grid frequency, the less visible is the grid in the sample plane, especially in thick tissues where aberrations can be important. Theoretical transverse resolution and depth of field are imposed by the microscope objective and are 0.36 and $1.45 \mu \mathrm{m}$, respectively, with the $40 \times$ water objective.

\subsection{Optimizing the Combination of the Modalities}

Simultaneously acquiring an FF-OCT image and an SIM image imposes several constraints, which must be optimized. The use of high NA objectives has two major advantages, as it enhances the collection of fluorescent photons and controls the thickness of the FF-OCT and fluorescence optical section, as described above.

The ability to control the optical section thickness from the spatial coherence offers additional versatility to our configuration, since the spectral bandwidth of the FF-OCT can be arbitrarily narrow to prevent the overlap between FF-OCT and fluorescence spectra. Ultimately, with high NA objectives, even a quasimonochromatic source can be used ${ }^{26,27}$ without altering the thickness of the optical section. Our setup configuration can be adjusted to use any visible wavelength for FFOCT, or fluorescence sources, according to the requirements of the application.

To prevent any spectral overlap between the two modalities, additional bandpass filters are inserted in front of both cameras (centered around 525 and $660 \mathrm{~nm}$, respectively). We overlay the images of the cameras by taking an image of the red illumination field diaphragm with both cameras by removing the filter in front of the sCMOS camera. Synchronization between the two cameras, the OCT and SIM piezoactuators, and potentially the illumination (to reduce illumination time and sample heating) is ensured by a DAQ card with $10 \mu$ s precision.

We should add that having a DF separating the FF-OCT and SIM paths out of the interferometer is not ideal since some of the fluorescence light is lost. However, we tried several configurations where a shortpass or longpass DF was in the sample arm, but we could never obtain a strong FF-OCT signal. Modeling and the shape of the interference patterns obtained in these first versions of the setup leads us to believe that the DF creates several optical paths due to its multiple coatings/reflection planes. Imaging fringes on a mirror will then give multiple, very low contrast rebounds, which will reduce the contrast of the main fringe pattern. However, if a dichroic mirror that does not affect FF-OCT signal can be found, the optimal configuration would be to insert this dichroic in the sample arm only.

To minimize this issue, we decided to use a 90:10 beamsplitter at the center of the interferometer and to mount the fluorescence detection part on the illumination side. Therefore, the illumination and fluorescence light are reflected at $90 \%$ by the beamsplitter, so $21 \%$ of the light used for fluorescence is lost instead of $75 \%$. Because the light in each path of the interferometer in FF-OCT has been transmitted and reflected once by the beamsplitter, the ratio $\sqrt{\left(\frac{R_{\text {Sample }}}{R_{\text {Ref }}}\right)}$ is conserved. The drawback is that we can collect only $9 \%$ of the light instead of $25 \%$ that we could have with a 50:50 beamsplitter. However, we chose the 90:10 configuration as we are often much more limited by the fluorescence intensity than the FF-OCT intensity, especially when using dynamic fluorescent probes.

Finally, our setup configuration requires two cameras, which implies a high cost, high complexity setup. However, the use of two cameras is unavoidable since FF-OCT and SIM require cameras with nearly opposite technical characteristics. FF-OCT requires fast cameras with large full well capacities, while fluorescence measurements require low noise measurements, enabling the detection of a small number of photons.

\subsection{Data Acquisition and Processing}

\subsubsection{Static FF-OCT}

Static FF-OCT images on zebrafish presented in Figs. 2(a) and 2(b) have been acquired with $10 \times, 0.3 \mathrm{NA}$ and $40 \times, 0.8 \mathrm{NA}$ objectives, respectively. The amplitude image at a given depth is computed from four direct images phase shifted by $\pi / 2$

$A(x, y)=\sqrt{\left[I\left(x, y, \phi_{0}\right)-I\left(x, y, \phi_{0}+\pi\right)\right]^{2}+\left[I\left(x, y, \phi_{0}+\frac{3 \pi}{2}\right)-I\left(x, y, \phi_{0}+\frac{\pi}{2}\right)\right]^{2}}$. 


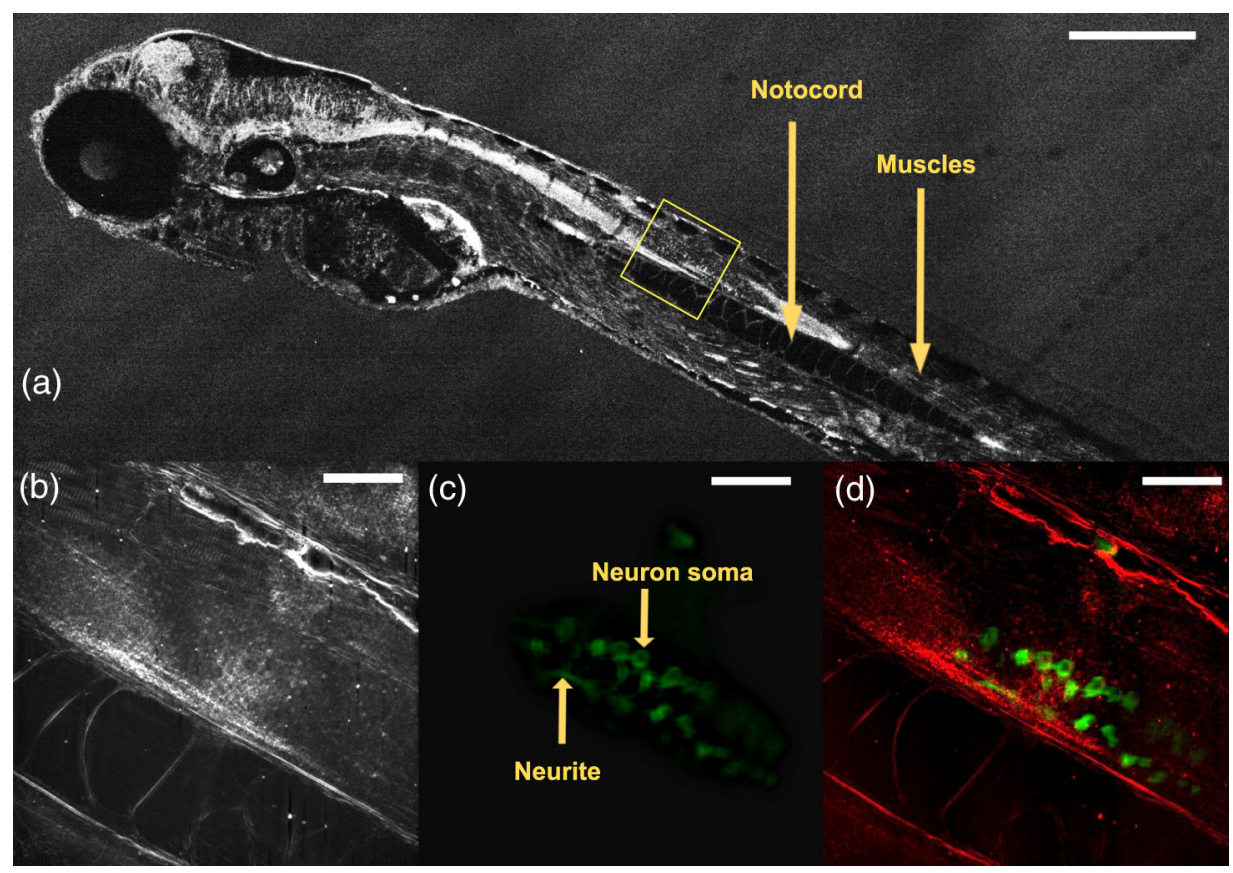

Fig. 2 Imaging of a Gal4 ${ }^{\text {s1020t }} /$ UAS: GFP zebrafish larva. (a) 10x wide-field full-field OCT image of the larva $100 \mu \mathrm{m}$ below the surface, first acquired with an FF-OCT commercial system, developed by LLTech. ${ }^{30}$ The main functional microstructures of the zebrafish can be identified. The scale bar represents $300 \mu \mathrm{m}$. (b-d) $40 \times 0.8 \mathrm{NA}$ images of the same larva then acquired with our multimodal setup, right after the imaging with the other microscope. The imaged area roughly corresponds to the yellow box in (a). (b) The FF-OCT image, (c) the SIM image, and (d) the overlay of (b) in red and (c) in green, illustrating the position of the larva motoneurons with respect to the notocord and spinalcord. All three scale bars represent $50 \mu \mathrm{m}$.

The images displayed in Figs. 2(a), 2(b), and 3(a) represent the average amplitude calculated over 10 consecutive images. The acquisition rate was 100 frames per second, enabling the acquisition of 25 amplitude images per second or 2.5 images per second for the 10-times-averaged amplitude image. The entire 3 -D volume of the zebrafish, representing about $4 \times 1 \times$ $0.3 \mathrm{~mm}^{3}$ was imaged in $2 \mathrm{~min}$ with the $10 \times$ objectives (not shown here). With these objectives, the field of view of a single image is $1.4 \times 1.4 \mathrm{~mm}^{2}$, and we had to stitch three transverse images to form one plane showing the entire fish. We scanned the sample in the $Z$ direction, with a $Z$-step of $3 \mu \mathrm{m}$, until the bottom of the dish. Here, the imaging depth is not an issue since zebrafish larvae are mostly transparent. The exposure time was $5 \mathrm{~ms}$, and the light exposure was centered within the rectangle of the piezoactuator displacement.

\subsubsection{Static SIM}

For optically sectioned fluorescent images shown in Figs. 2(c) and 3(b), we also acquired four consecutive images to exclude out of focus fluorescence. The equation is similar to Eq. (1), but the phase change represents a change in the phase of the grating pattern. We used an exposure time of $199 \mathrm{~ms}$, enabling an acquisition rate of 5 direct frames per second or 1.25 optically sectioned images per second.

\subsubsection{Dynamic FF-OCT}

In dynamic FF-OCT, we are interested in the temporal phase fluctuations of the tissue. These phase fluctuations are captured either from the direct images or from phase images with a similar effect, but we usually prefer to use direct images to reduce mechanical vibrations caused by the piezoactuator motion. ${ }^{7}$ Therefore, we stopped the modulation of the piezoelement, i.e., of the optical path. Due to the low coherence of the setup, we are only sensitive to phase changes that happen at a given depth inside the coherence gate of the microscope. In Fig. 3(c), we acquired 100 consecutive direct images and computed the standard deviation (STD) in groups of 10 consecutive images, before computing the average of the 10 resulting images. We wish to emphasize the fact that computing the STD on the direct images cancels the incoherent light that does not produce interference. Therefore, the image obtained corresponds to fluctuations happening at a given depth, similar to what is obtained by modulating the piezoposition.

Even though the final image is obtained in $1 \mathrm{~s}$, we are probing phase fluctuations caused by intracellular displacements that are occurring in the 10 to $100 \mathrm{~Hz}$ range.

\subsubsection{Waves propagation imaging}

In the experiment presented in Sec. 3.3, we imaged mechanical and calcium waves propagating close to the surface of a heart wall. The FF-OCT acquisition scheme is similar to that described for dynamic FF-OCT. We imaged 1000 consecutive direct images and looked for the phase fluctuations caused by the propagation of mechanical elastic waves in the tissue. Once again, we emphasize that it is crucial to have an interferometer to measure these mechanical waves. First, due to its high axial sensitivity, our microscope is able to measure mechanical deformations smaller than $100 \mathrm{~nm}$. Additionally, 
because of the low coherence, the phase deformations can only be measured if the deformation occurs within the coherence volume.

To enhance the signal-to-noise ratio, we computed the STD over five consecutive images and performed the calculation by applying a sliding window. This means that we computed 995 images of STD calculated with four identical images for two consecutive frames. The STD highlights the averaged propagating wavefront between the five images. Even though four images are identical between two consecutive frames with the sliding window, the averaged wavefront propagated during a time equal to a single frame time. Using such a sliding window enables the acquisition of propagating phenomena at an apparent acquisition rate of 100 frames per second, but it reduces the displacement sensitivity and would logically fail at detecting changes occurring with frequencies higher than $20 \mathrm{~Hz}$.

For the fluorescence measurement in the wave imaging experiment, we decided to remove the grating in the illumination path to increase the light power, so we could decrease the exposure time and increase the acquisition rate accordingly. We therefore lost the sectioning ability of the fluorescence part. However, even though the background is stronger, we are quite confident that the fluorescence increase (related to calcium uptake) comes from the imaged plane because the increase is localized spatially along the fiber that can be observed in the direct image. If the calcium uptake was coming from an outof-focus structure, it would correspond to an increase of the fluorescence background.

By removing the grating, we succeeded in reducing the exposure time to $49 \mathrm{~ms}$ and to imaging at 20 frames per second. This is fast enough to observe a fluorescence increase related to calcium wave propagation. In Fig. 4(b), we overlaid the fluorescence difference in green (after subtracting the mean) to the average fluorescence intensity in gray. In Fig. 4(e), we plotted the temporal profile of the fluorescence difference along the red line, and we subtracted the temporal average at each pixel again.

Video 1 is the movie of the temporal evolution of the same heart wall acquired with the two modalities. The left image represents the evolution of the STD on five consecutive images. The right image represents the evolution of the fluorescence change. In this video, we did not perform a sliding window to match the acquisition rate of the two modalities.

\subsection{Sample Preparation}

\subsubsection{Zebrafish larvae}

Experiments were performed on zebrafish larvae at 5 days post fertilization (dpf) following procedures approved by the Institutional Ethics Committee Darwin in the Institut du Cerveau et de la Moelle épinière (ICM). Zebrafish larvae grew in an incubator at $28.5^{\circ} \mathrm{C}$ until $5 \mathrm{dpf}$. Shortly before the experiment, the larvae were laterally mounted in $1.5 \%$ low melting point agarose and paralyzed by injecting $0.5 \mathrm{nl}$ of $0.5 \mathrm{mM}$ $\alpha$-bungarotoxin in the ventral axial musculature. The experiments were performed at room temperature. Gal4 ${ }^{s 1020 t} / \mathrm{UAS}$ : green fluorescent protein (GFP) transgenic larvae were used, in which GFP is expressed in spinal cord motoneurons and in neurons in the thalamus.

\subsubsection{Retinae experiments}

For the fluorescence and dynamic FF-OCT experiments described in Sec. 3.2, fresh macaque retina explants were imaged. All animal manipulation was approved by the Quinze Vingts National Ophthalmology Hospital and regional review board (CPP Ile-de-France V), and was performed in accordance with the ARVO Statement for the Use of Animals in Ophthalmic and Vision Research. Anterior segments were removed, and either the full thickness retina was imaged with the retinal pigment epithelium (RPE) and choroid intact or the retinas were dissected at the level of the photoreceptor outer segments, so the RPE and choroid were excluded from the images. Retinal samples were flatmounted either directly in the FFOCM sample holder or adhered to a membrane support for transfer to the FFOCM sample holder, immersed in $\mathrm{CO}_{2}$-free neurobasal medium, and gently immobilized under a thin transparent microporous membrane (PET membranes, BdBiosciences).

The retinal ganglion cells were labeled in vitro by particlemediated acute gene transfer of GFP. A Helios gene gun system (Bio-Rad, Hercules, California) was used for particle-mediated acute gene transfer in macaque retinal explants, similarly to what has been previously described. ${ }^{31}$ Briefly, 10 -mg gold microcarriers $(1.6 \mu \mathrm{m}$; Bio-Rad) were coated with $20 \mu \mathrm{g}$ of a plasmid encoding CMV:CatCh-GFP in an AAV backbone in 3.2-mL ethanol solution and loaded into Tefzel tubing (Saint Gobain, Forge, Pennsylvania) using the gene gun Tubing Prep Station (Bio-Rad). The gene gun barrel was held $5 \mathrm{~mm}$ above the retinal explant (ganglion cell side facing the barrel), and the plasmid bullets were propelled at a pressure of 110 psi. The explants were incubated at $37^{\circ} \mathrm{C}$ and $5 \% \mathrm{CO}_{2}$ for 1 day postgene transfer and were imaged in Neurobasal-A medium (ThermoFisher Scientific).

\subsubsection{Heart experiments}

Rats were housed individually with free access to food and water and maintained in a temperature-controlled environment on a 12-h light/dark cycle. Experiments were performed in agreement with the institutional guidelines for the use and care of animals and in compliance with national and international laws and policies (Council directives no. 87-848, October 19, 1987, Ministère de l'Agriculture et de la Forêt, Service Vétérinaire de la Santé et de la Protection Animale). Shortly after death, the beating heart was dissected and the interventricular septum was extracted, giving access to the region where the atrioventricular bundle is located. This region exhibits a spontaneous activity elicited by pacemaker cells and is involved in heart beat regulation. ${ }^{32}$ The interventricular septum is soaked in a $10-\mu \mathrm{M}$ Fluo-4, AM, cell permeant (ThermoFisher Scientific) Ringer's solution, containing ATP and glucose sources, for $45 \mathrm{~min}$ at $36.5^{\circ} \mathrm{C}$. The tissue is then washed twice in Ringer's solution, and finally inserted under the microscope for the imaging.

The heart beat can be maintained up to a few hours after the animal death, as long as the heart is kept in physiological conditions and is well supplied in ATP and oxygen. ${ }^{33}$ Most heart donations are made from beating heart donors, in which case the heart can be kept beating for up to a few hours after the donor's death. ${ }^{34}$ This activity is the consequence of the autonomous activity of the heart, so we expect it to last for a bit longer in the interventricular septum, which is one of the centers of this autonomous activity. 


\section{Experimental Results}

\subsection{Static FF-OCT and Static SIM}

First, we demonstrate the interest of our system for combining structural information with specific molecular contrast, similar to what has been demonstrated previously. ${ }^{20,22,35}$ Figure 2 shows such a combination in 5 days postfertilization zebrafish larvae with fluorescent motoneurons. Figures 2(a) and 2(b) show en face views of the structural contrast of a zebrafish larva at different magnifications.

The interest of the FF-OCT image is to image the microstructures of the zebrafish larva at a given depth. However, if the muscles and the notocord are clearly visible on the FF-OCT image, we can only guess at the presence of some cells corresponding to small white round objects in the image. Among these cells, there is no information about their nature or their function. To identify the cells, we imaged the same zebrafish, which is from transgenic line exhibiting GFP-fluorescent motoneurons along the larva spinal cord. Figure 2(c) illustrates an SIM image obtained in such a zebrafish larva, using the fluorescence part of our setup.

Combining the two modalities allows us to see the organization of these motoneurons with respect to the surrounding tissue of the fish; in particular, we are able to see that some of these neurons have projections either onto the notocord or onto the spinal cord.

\subsection{Dynamic FF-OCT and Static SIM}

To further illustrate the interest of our system for identifying specific structures visible with dynamic-FF-OCT using fluorescence, we imaged a macaque retina, labeled via gene gun to tag ganglion cells inside the retina. The gene gun technique produces a sparse labeling. ${ }^{31}$ Due to fluorescence imaging, we were able to confirm that the cells we were imaging were indeed ganglion cells.

Here, the imaging plane is in between the ganglion cell layer and the inner plexiform layer, and a strong signal from stationary fibers inside the inner plexiform layer dominates the static FF-OCT image, as shown in Fig. 3(a). However, using the temporal fluctuations of the signal and displaying the average image over 10 STD images calculated over 10 consecutive FF-OCT direct images, we could attenuate the signal from the collagen and reveal the cells at this depth, as shown in Fig. 3(c). However, two populations of cells can be observed in the image, as expected, since the ganglion cell layer is mainly composed of ganglion cells and displaced amacrine cells. ${ }^{36}$ The overlay performed in Fig. 3(d) shows that the ganglion cell labeled with fluorescence perfectly matches one of the cells at the center of the D-FF-OCT. In accordance with the literature describing ganglion cell morphology and distribution, ${ }^{36}$ we postulate that the dark central region corresponds to the nucleus, while the elongated bright surround is the cytoplasm. It appears that the axon hillock can also be observed in D-FF-OCT, as it

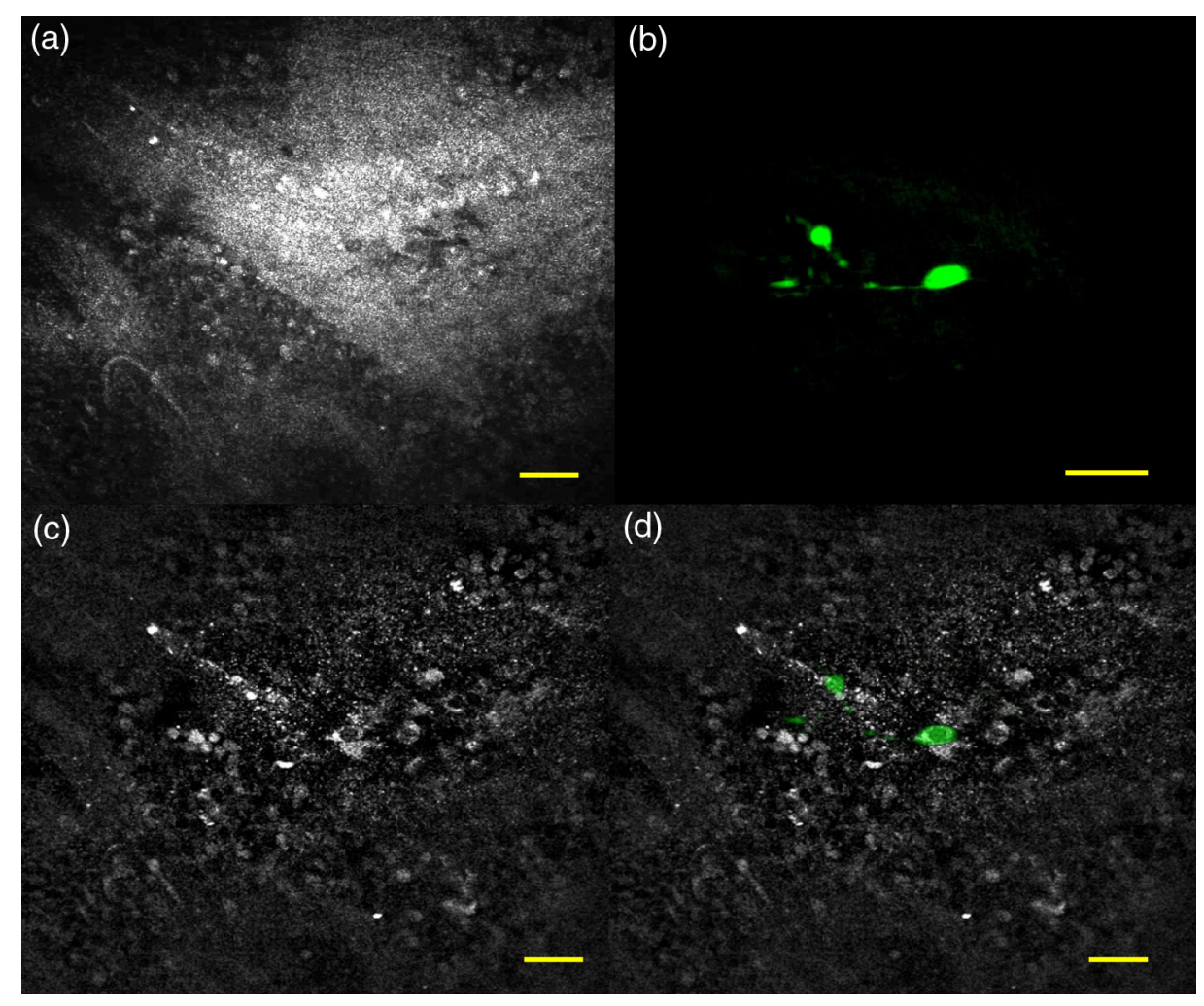

Fig. 3 Multimodal SIM/dynamic FF-OCT of a macaque retina. (a-d) Show the ganglion cell layer of a fresh macaque retina revealed by three different modalities acquired simultaneously with our microscope. (a) The FF-OCT image and signal from ganglion cells are masked by collagen signal. A sparse labeling of ganglion cells has been performed on this retina, and the corresponding SIM image is displayed in (b). (c) The same field of view imaged with dynamic FF-OCT, which removes the signal from the static collagen fibers to reveal several cells. The D-FF-OCT also reveals intracellular details. Finally, (d) is the overlay between the dynamic FF-OCT image and the SIM image, revealing a perfect overlap between one of the ganglion cells and one cell revealed by dynamic FF-OCT. All the scale bars represent $35 \mu \mathrm{m}$. 
matches the beginning of the ganglion cell axon labeled with fluorescence. The other rounded cells with lower intracellular contrast might then be amacrine cells. This is consistent over many more FF-OCT and D-FF-OCT images of the retinal ganglion cell layer, where the two same populations are observed. ${ }^{22}$

In this figure, only one ganglion cell is labeled with fluorescence due to the low success rate of the gene gun, producing a sparse labeling. The two smaller hot fluorescence spots are much likely buddings along the ganglion cell neurites, which we do not necessarily see in D-FF-OCT. We can add that we are now trying to measure the ratio of ganglion cells that D-FF-OCT is able to reveal in the ganglion cell layer, but it is probably not $100 \%$.

\subsection{Dynamic FF-OCT and Dynamic Fluorescence}

Finally, we imaged dynamic processes in both FF-OCT and fluorescence to illustrate the importance of synchronizing the two modalities. Video 1 and Fig. 4 show one result of imaging a fresh beating rat heart interventricular septum. Video 1 displays the evolution of the FF-OCT signal on the left side. The evolution of the STD calculated over five direct images without piezomodulation is presented. On the rightside, the evolution of the fluorescence signal is displayed. We can identify mechanical and calcium waves propagating in the tissue. Figure 4 presents the same data set as a still image.
Figures 4(a) and 4(c) present the mechanical wave observed with FF-OCT, where we can see a propagating wavefront in (a) and typical tilted lines in the spatiotemporal profile in (c) This profile allows calculation of the wave speed of around $100 \mu \mathrm{m} . \mathrm{s}^{-1}$, which is too slow to be a mechanical wave, ${ }^{10}$ especially in a stiff tissue such as the heart. Therefore, with the FF-OCT information only, it is complicated to understand what this wave corresponds to.

On the other hand, the fluorescence information shows slow calcium uptakes and slow calcium propagation happening simultaneously with the mechanical waves. Figures 4(b) and 4(e) show calcium propagation in the tissue and its spatiotemporal profile along the red line of (b), respectively. Interestingly, the measured propagation speed of the calcium, measuring the averaged slope in (e), is also very close to $100 \mu \mathrm{m} . \mathrm{s}^{-1}$. Interestingly, the overlay of the spatiotemporal profiles, presented in (d), shows a good agreement between the propagation speeds of the two waves.

For this experiment, we used wide-field fluorescent measurement instead of SIM as we imaged close to the surface of the tissue, where there is less need for optical sectioning, and we wanted to maximize the acquisition speed and the signal magnitude for this particular application.

To conclude this subsection, our multimodal setup enables simultaneous imaging of the propagation of a mechanical wave using FF-OCT and the propagation of a calcium wave using
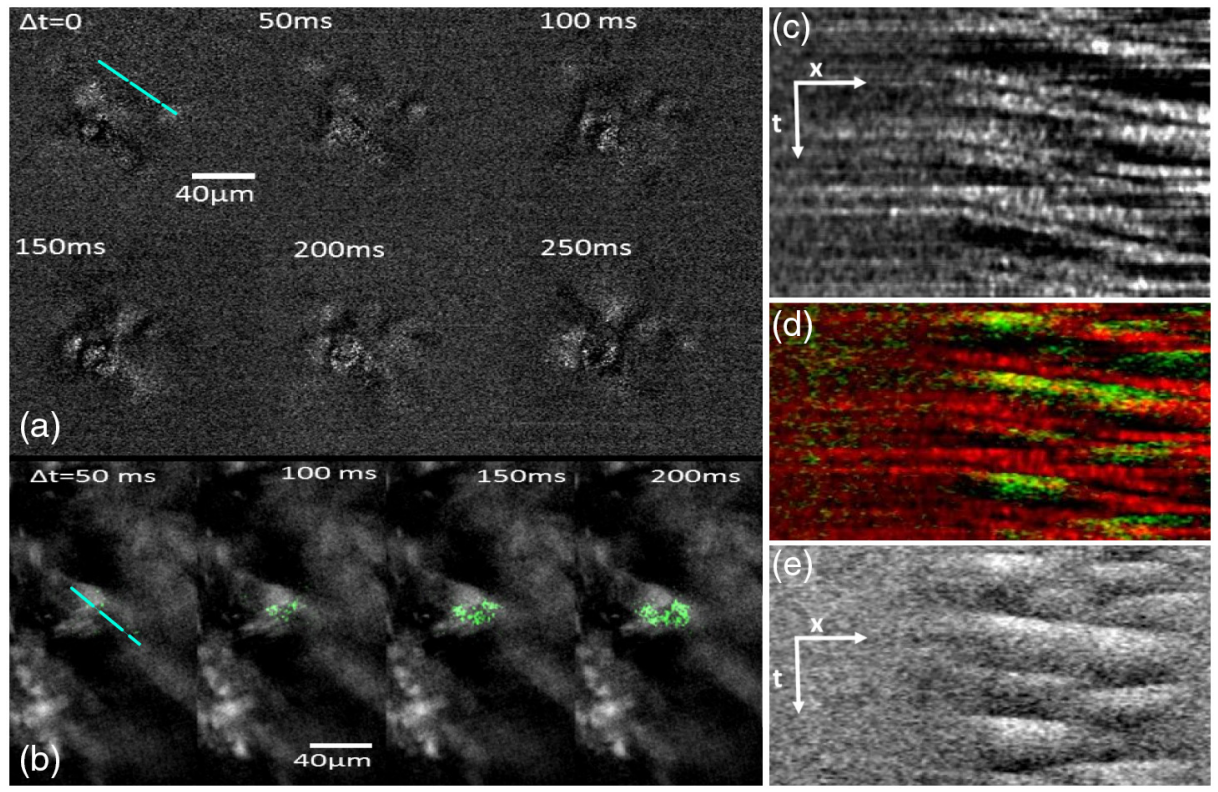

Fig. 4 Dynamic imaging of mechanical wave and calcium waves in a beating heart. (a-e) Show the result of imaging a beating section of a rat heart using FF-OCT and fluorescence microscopy. Video 1 (QuickTime, 5.25 MB [URL: http://dx.doi.org/10.1117/1.JBO.22.2.026004.1]) presents the raw data, from which (a-e) have been computed. (a) The propagation of a mechanical wave observed over time along a fiber. The corresponding fiber is masked with the red line in the first image. The images are the results of the STD of five consecutive images to enhance the signal-to-noise ratio. The scale bar represents $40 \mu \mathrm{m}$. (c) A temporal profile along the cyan dashed line presented in (a). This is the result of a sliding STD to keep the temporal resolution. On the right of (c), the profile exhibits clear parallel tilted lines, characteristic of propagating waves. The scale bars represent $13 \mu \mathrm{m}$ and $550 \mathrm{~ms}$, respectively. The slope of the lines can give us the wave propagation speed of about $100 \mu \mathrm{m} . \mathrm{s}^{-1}$. (b) The initiation and propagation of calcium waves imaged from the Fluo-4, a calcium fluorescent indicator, response. To enhance the signal-to-noise ratio, the fluorescence image has been superimposed on the fluorescence variation $\Delta F$, in green, at different times. The scale bar represents $40 \mu \mathrm{m}$ and is valid for all five images of the panel. (e) The temporal profile along the cyan dashed line of (b). This fiber corresponds to the fiber along which the mechanical wave propagates, as illustrated in $(a, b)$. The profile represents the fluorescence difference $\Delta F$ across the fiber. Finally, (d) the overlay of $(c, e)$. 
fluorescence. Due to the simultaneous nature of the measurements, we demonstrate that the mechanical waves propagating in the sample are linked with biological activity in the tissue, i.e., the calcium wave, which is known to cause fiber contraction. ${ }^{37}$ It is particularly critical here in an ex vivo tissue to acquire both pieces of information at the same time. Indeed, since the tissue is altered, its activity can be rapidly decreasing. Therefore, with a sequential acquisition multimodal system, by the time a movie was captured with one modality, the activity may have stopped before switching to the other. Also, as we are looking for waves, switching between modalities from one frame to the next would significantly lower the acquisition speed, meaning the wave propagation might be undetectable.

\section{Discussion and Conclusion}

In this study, we present a new approach able to perform multimodal static and dynamic FF-OCT and SIM. This combination enables acquisition of images from both modalities simultaneously. We show that using high NA objectives enables optimization of the characteristics of this configuration since more fluorescence is collected, and the optical sectioning used in FF-OCT is controlled by the spatial coherence mainly imposed by the NA of the objective. Therefore, we can obtain fine axial sections with a low spectral bandwidth light, meaning that the FF-OCT spectrum does not overlap with the fluorescence spectrum. Our system exhibits good transverse resolution of around $500 \mathrm{~nm}$ and an axial sectioning close to $4 \mu \mathrm{m}$.

We demonstrate the interest of our setup to efficiently combine contrast from the three modalities and to identify structures observed with FF-OCT and dynamic FF-OCT. Using a multimodal microscope instead of a sequential imaging on two separate microscopes is important to colocalize small structures and to preserve samples from degradation, as it decreases acquisition and operational times. Acquisition time can be further decreased by using our simultaneous design, which enables the following of biochemical and structural changes with unprecedented spatial and temporal resolutions.

Finally, in Fig. 4 and Video 1, we emphasized the advantage offered by the simultaneity of the two measurements. We could measure the copropagation of a slow mechanical wave and its corresponding calcium wave in a beating heart.

Ultimately, such a simultaneous combination is particularly important for observing dynamic changes in the microstructure of a tissue imaged with FF-OCT and for linking these changes with modifications of the biochemical properties of the surrounding medium due to electrical activation, a change in $\mathrm{pH}$, or in ATP concentration, detectable by fluorescent probes.

\section{Disclosures}

No conflicts of interest, financial or otherwise, are declared by the authors.

\section{Acknowledgments}

The authors want to thank Charles Edouard Leroux for his help in the design of the microscope and Clément Apélian for discussions and advice on dynamic full-field OCT and for tissues preparation. We are grateful for the help of Kevin Fidelin and Claire Wyart for discussion and preparation of the zebrafish experiments. We also acknowledge the important help from Kate Grieve and Abhishek Sengupta for retina preparation and fluorescence labeling. We thank Charlie Demé né and Jérome Mairesse for heart dissections, preparations, and advice on the heart experiments. We finally express our gratitude to Thu-Mai Nguyen and especially Kate Grieve for the numerous manuscript revisions. This work was supported by grants from the European Research Council SYNERGY Grant scheme (HELMHOLTZ, ERC Grant Agreement \# 610110; European Research Council, Europe) and LABEX WIFI (Laboratory of Excellence ANR-10-LABX-24) as part of the French Program "Investments for the Future" under reference ANR-10-IDEX0001-02 PSL.

\section{References}

1. D. Huang et al., "Optical coherence tomography," Science 254(5035), 1178-1181 (1991).

2. W. Drexler and J. G. Fujimoto, Optical Coherence Tomography Technology and Applications, Springer, Berlin; New York (2008).

3. E. Beaurepaire et al., "Full-field optical coherence microscopy," Opt. Lett. 23(4), 244-246 (1998).

4. A. Dubois et al., "Ultrahigh-resolution full-field optical coherence tomography," Appl. Opt. 43(14), 2874-2883 (2004).

5. J. Ben Arous et al., "Single myelin fiber imaging in living rodents without labeling by deep optical coherence microscopy," J. Biomed. Opt. 16(11), 116012 (2011).

6. S. Mukherjee et al., "Modified full-field optical coherence tomography: a novel tool for rapid histology of tissues," J. Pathol. Inf. 2(1), 28 (2011).

7. C. Apelian et al., "Dynamic full field optical coherence tomography: subcellular metabolic contrast revealed in tissues by interferometric signals temporal analysis," Biomed. Opt. Express 7(4), 1511-1524 (2016).

8. J. Lee et al., "Dynamic light scattering optical coherence tomography," Opt. Express 20(20), 22262-22277 (2012).

9. C.-E. Leroux et al., "Intracellular dynamics measurements with full field optical coherence tomography suggest hindering effect of actomyosin contractility on organelle transport," Biomed. Opt. Express 7, 4501-4513 (2016).

10. T.-M. Nguyen et al., "Visualizing ultrasonically induced shear wave propagation using phase-sensitive optical coherence tomography for dynamic elastography," Opt. Lett. 39, 838 (2014).

11. T.-W. Chen et al., "Ultrasensitive fluorescent proteins for imaging neuronal activity," Nature 499(7458), 295-300 (2013).

12. K. Gee et al., "Chemical and physiological characterization of fluo-4 $\mathrm{Ca}^{2+}$-indicator dyes," Cell Calcium 27, 97-106 (2000).

13. M. S. Siegel and E. Y. Isacoff, "A genetically encoded optical probe of membrane voltage," Neuron 19(4), 735-741 (1997).

14. H. Imamura et al., "Visualization of ATP levels inside single living cells with fluorescence resonance energy transfer-based genetically encoded indicators," Proc. Natl. Acad. Sci. U. S. A. 106(37), 15651-15656 (2009).

15. M. A. A. Neil, R. Jukaitis, and T. Wilson, "Method of obtaining optical sectioning by using structured light in a conventional microscope," Opt. Lett. 22, 1905 (1997).

16. J. Mertz, Introduction to Optical Microscopy, Vol. 138, Roberts and company, Greenwood Village, Colorado (2010).

17. M. G. L. Gustafsson, "Nonlinear structured-illumination microscopy: Wide-field fluorescence imaging with theoretically unlimited resolution," Proc. Natl. Acad. Sci. U. S. A. 102, 13081-13086 (2005).

18. E. Beaurepaire et al., "Combined scanning optical coherence and twophoton-excited fluorescence microscopy," Opt. Lett. 24(14), 969-971 (1999).

19. S. Yuan et al., "Co-registered optical coherence tomography and fluorescence molecular imaging for simultaneous morphological and molecular imaging," Phys. Med. Biol. 55, 191-206 (2010).

20. E. Auksorius et al., "Dual-modality fluorescence and full-field optical coherence microscopy for biomedical imaging applications," Biomed. Opt. Express 3, 661-666 (2012).

21. F. Harms et al., "Multimodal full-field optical coherence tomography on biological tissue: toward all optical digital pathology," Proc. SPIE 8216, 821609 (2012).

22. K. Grieve et al., "Appearance of the retina with full-field optical coherence tomography," Invest. Ophthalmol. Vis. Sci. 57, OCT96-OCT104 (2016). 
23. H. Makhlouf et al., "Simultaneous optically sectioned fluorescence and optical coherence microscopy with full-field illumination," Opt. Lett. 37, 1613 (2012).

24. I. Abdulhalim, "Spatial and temporal coherence effects in interference microscopy and full-field optical coherence tomography," Ann. Phys. 524, 787-804 (2012).

25. M. Quinten, Optical Properties of Nanoparticle Systems: Mie and Beyond, Wiley-VCH Verlag \& Co. KGaA, Weinheim, Germany (2010).

26. A. Dubois et al., "Phase measurements with wide aperture interferometers," Appl. Opt. 39(14), 2326-2331 (2000).

27. A. Safrani and I. Abdulhalim, "Ultrahigh-resolution full-field optical coherence tomography using spatial coherence gating and quasi-monochromatic illumination," Opt. Lett. 37(4), 458-460 (2012).

28. B. Karamata et al., "Spatially incoherent illumination as a mechanism for cross-talk suppression in wide-field optical coherence tomography," Opt. Lett. 29(7), 736-738 (2004).

29. P. Xiao, M. Fink, and A. C. Boccara, "Full-field spatially incoherent illumination interferometry: a spatial resolution almost insensitive to aberrations," Opt. Lett. 41, 3920-3923 (2016).

30. E. Dalimier, "Commercial system lltech," 2013, http://www. lltechimaging.com/products-applications/products/ (January 2017).

31. S. Moritoh et al., "Diversity of retinal ganglion cells identified by transient GFP transfection in organotypic tissue culture of adult marmoset monkey retina," PLoS One 8, e54667 (2013).

32. A. B. Arrenberg et al., "Optogenetic control of cardiac function," Science 330, 971-974 (2010).

33. "Vulgarization article describing heart beating after death," TheScienceBehind, 2011, http://science-behind.blogspot.fr/2011/03/ why-hearts-continue-to-beat-after-death.html (December 2016).

34. N. Zamperetti, R. Bellomo, and C. Ronco, "Defining death in non-heart beating organ donors," J. Med. Ethics 29(3), 182-185 (2003).

35. K. Grieve et al., "Three-dimensional structure of the mammalian limbal stem cell niche," Exp. Eye Res. 140, 75-84 (2015).
36. M. Kalloniatis and C. Luu, "Visual acuity," in Webvision: The Organization of the Retina and Visual System, H. Kolb, E. Fernandez, and R. Nelson, Eds., University of Utah Health Sciences Center, Salt Lake City, Utah (1995).

37. J. Pinnell, S. Turner, and S. Howell, "Cardiac muscle physiology," Contin. Educ. Anaesth. Crit. Care Pain 7, 85-88 (2007).

Olivier Thouvenin is currently working at the Institut Langevin (Paris, France), ESPCI Paris, PSL Research University as a PhD student. He is part of a doctoral program within Paris Diderot (Paris 7) University and the Center for Research and Interdisciplinarity (CRI Paris). He received his MSc degree from ENS Lyon (Lyon, France). His current research interests include OCT, quantitative phase imaging, and neurosciences. He is notably interested in understanding mechanical cues coupled with neuronal activity.

Mathias Fink is a professor at the Institut Langevin, ESPCI Paris, PSL Research University. He received his $\mathrm{PhD}$ in solid-state physics in 1970 and received the doctorates-sciences degree in 1978 from Paris University. His current research interests include medical ultrasonic imaging, ultrasonic therapy, nondestructive testing, telecommunications, seismology, active control of waves, wave coherence in multiply scattering media, and time reversal in physics. He holds 28 patents, and he has published more than 300 articles.

Claude Boccara is dean of research at Institut Langevin, ESPCI Paris, PSL Research University. He has developed new microscopies to increase depth and lateral resolution. Recently, ultimate measurements have found new fields of application going from detection of gravitational waves to $3-D$ imaging though scattering media. He has published more than 300 scientific articles (ISI/Boccara $A^{*}$ or Boccara C) in international journals. In 2007, he founded the startup LLTech devoted to medical imaging and diagnostics. 